\title{
US inflation with trace and simultaneous fear conditioning
}

\author{
JACK EDWARD SHERMAN \\ University of Colorado, Boulder, Colorado 80302
}

\begin{abstract}
Two experiments with rat subjects in a conditioned punishment paradigm are reported. These experiments attempted to determine if the events entering into association with the CS following conditioning with informative (forward) and noninformative (simultaneous) CSs were comparable. In Experiment 1, exposure to intense shock alone following trace (ISI $=10 \mathrm{sec}$ ) conditioning with moderate shock enhanced the suppressive effects of a 2 -sec CS. A similar manipulation following explicitly unpaired CS-US presentations (ISI $=2 \mathrm{~min}$ ) had no effect. These data were taken as evidence that the CS and US were associated during trace conditioning. Experiment 2 showed that exposure to intense shock following simultaneous conditioning also enhanced suppression to the CS. These results suggested that simultaneous and forward conditioning procedures yield similar forms of associative learning.
\end{abstract}

The recent demonstrations of excitatory simultaneous fear conditioning (Heth \& Rescorla, 1973; Mahoney \& Ayres, 1976; Sherman \& Maier, 1978) pose a problem for informational views of classical conditioning (Egger \& Miller, 1962; Kamin, 1969; Mackintosh, 1973, 1975). This is because, unlike forward procedures in which the conditioned stimulus (CS) signals the subsequent occurrence of the unconditioned stimulus (US), the simultaneous CS is redundant and noninformative with respect to US occurrence. The present investigation searches for qualitative differences in the associative learning which mediates fear conditioning with informative (forward) and noninformative (simultaneous) CSs. If simultaneous conditioning is found to be a product of a different associative process than that underlying forward conditioning, informational views of conditioning might thereby be reconciled.

The feasibility of addressing this issue is based on Rescorla's $(1973,1974)$ recent research in fear conditioning which strongly suggests that different events enter into association with the CS as a consequence of the specific conditioning procedure employed. This is so even though qualitatively similar indices of conditioning are observed. Rescorla's work suggests that in first-order fear conditioning an association is formed between the CS and US (shock). However, with second-order fear conditioning in which the reinforcer is a previously conditioned CS, the second-

This research was supported by Grant MH 26827 to Steven F. Maier. This study is based on part of a dissertation thesis submitted in partial fulfillment of the requirements for the $\mathrm{PhD}$ degree at the University of Colorado by Jack E. Sherman. Thanks are due to Steven Maier and Eric Holman for providing critical comments on an earlier version of this manuscript. Reprint requests should be sent to J. E. Sherman, Department of Psychology, University of California, Los Angeles, California 90024. order $\mathrm{CS}\left(\mathrm{CS}_{2}\right)$ is thought to be directly associated with the fear reaction evoked by the first-order CS. Traditionally, the former type of association is termed S-S (for stimulus-stimulus) learning and the latter S-R (for stimulus-response) learning.

Rescorla's procedure for demonstrating that firstorder conditioning is of an S-S nature is based on a paradigm first suggested by Rozeboom (1958). Rescorla (1974) found that if rats were presented a severe shock alone after CS pairings with a moderate shock, an enhanced degree of conditioned suppression to the CS was obtained. Presumably, experience with severe shock modified the representation, or memory, of the US by "inflating" its value. Subsequently, when the CS was presented alone it presumably activated, or evoked, the inflated representation of the US, resulting in a stronger fear reaction and hence more suppression.

In second-order conditioning, this same US manipulation had no effect on the suppressive effects of $\mathrm{CS}_{2}$. Rescorla argued that the events entering into association with $\mathrm{CS}_{2}$ must therefore be of a different nature. He suggested that the response evoked by the first-order CS was the most likely candidate. This pattern of results, in which a first-order CS is subject to US representation manipulations whereas a secondorder CS is not, is of considerable generality (Rescorla, 1977).

It may be argued that the excitatory conditioning obtained with both simultaneous and second-order conditioning is preasymptotic and does not yet reflect learning which is based on the informational relationship of the CS to the US. Consistent with this notion, Heth (1976) and Sherman and Maier (1978) found that increasing the number of excitatory conditioning trials decreased the excitatory effects of the CS. Similarly, Rescorla (1972) not only observed 
decreases in the excitatory effects of a second-order CS with increased trials but also observed the eventual development of inhibition, since shock never followed $\mathrm{CS}_{2}$ in his second-order procedures. Thus, the asymptotic performance observed with both these conditioning procedures is consistent with an informational view of conditioning. These parallels in the course of acquisition of simultaneous and secondorder conditioning suggest that the associative learning mediating these preasymptotic, noninformational effects may be similar. Therefore, analogous to second-order conditioning, simultaneous conditioning may also fail to be affected by procedures designed to manipulate the representation of the US.

The present experiments address whether a postconditioning US-inflation manipulation influences fear conditioning following simultaneous as well as forward conditioning procedures. It is difficult to assess simultaneous fear conditioning using the standard conditioned emotional response (CER) techniques because the $\mathrm{CS}$ in simultaneous conditioning is typically very brief. The most frequently used measure of fear conditioned during simultaneous conditioning has been an assessment of the CS's ability to punish appetitive instrumental responding (conditioned punishment). However, Rescorla's USinflation experiment studied CER conditioning with a 2-min CS. Thus, the present study first requires a demonstration of a US-inflation effect with forward conditioning using a brief $\mathrm{CS}$ and conditioned punishment measurement procedure. Experiment 1 attempts to demonstrate US-inflation effects following conditioning with a $2-\mathrm{sec}$ trace CS and a $10-\mathrm{sec}$ interstimulus interval (ISI).

\section{EXPERIMENT 1}

Method
Subjects
The subjects were 48 male Sprague-Dawley albino rats obtained
from the Holtzman suppliers in Madison, Wisconsin. The rats
were approximately $90-100$ days old at the start of the experiment,
and were maintained at $80 \%$ of their free-feeding weights.

\section{Apparatus}

The rats were run in eight similar experimental chambers, which were individually housed in sound-attenuating enclosures, each equipped with a ventilating fan. The experimental chambers were made of Plexiglas and aluminum; the floor consisted of stainless steel rods, through which scrambled shock could be presented. A retractable lever was attached to the front metal wall of each chamber next to the food tray. Leverpresses were reinforced by the presentation of $.045-\mathrm{g}$ P. J. Noyes pellets. A detailed description of these chambers is presented elsewhere (Sherman \& Maier, 1978)

The CS consisted of the simultaneous onset of light and tone. The light was provided by a $28-\mathrm{V}$ No. 1821 bulb mounted on the far wall of the sound-attenuating chamber; the chamber was otherwise dark. The $1,800-\mathrm{Hz}$ tone was delivered through an 8 -ohm $11.43-\mathrm{cm}$ high-fidelity loudspeaker (Jensen Sound Laboratories) mounted on the center of the Plexiglas ceiling. This signal increased the background noise of each chamber (the mean was $71 \mathrm{~dB}$ ) by $5 \mathrm{~dB}$ measured on the A scale of a General Radio audiometer, Model 1565-b (re: $20 \mu \mathrm{N} / \mathrm{m}^{2}$ ). Measurements of sound pressure level were taken in the approximate region of the rat's head when leverpressing. White noise was delivered in the experimental room to mask extraneous sounds.

\section{Procedure}

Leverpress and baseline training. To facilitate leverpress training, several Noyes pellets were left on the lever and in the food tray. Each subject was permitted to earn 100 pellets on a continuous schedule of reinforcement. On each of the next 10 days, the rats received $40-\mathrm{min}$ sessions in which leverpressing produced food pellets according to a constant probability variable-interval (VI) 1-min schedule (Catania \& Reynolds, 1968). With the exception of conditioning and postconditioning US exposure sessions, all sessions began with eight pellets present in the food tray. This was done to facilitate recovery of baseline following the shockalone treatment.

Conditioning and postconditioning US exposure. After baseline training, rats were matched for response rates and assigned to one of six groups $(n=8)$. Three of these groups received paired (P) CS-US presentations with an ISI of $10 \mathrm{sec}$, and the other three groups received unpaired (U) presentations with an ISI of $120 \mathrm{sec}$.

Eight presentations of CS and US were given for each of two successive sessions. The CS was a 2 -sec tone-light compound, and the US was a 2-sec .4-mA shock. CS presentations occurred at the same relative time during each session for all groups. The intertrial intervals (ITI) were arranged according to a randomly selected rectangular distribution of 4-, 5-, 6-, and 7-min intervals daily.

During each of the next two successive sessions, all groups received eight shock-alone presentations. For each of the three groups receiving paired or unpaired CS-US presentations, shock intensity was $0, .4$, and $1.6 \mathrm{~mA}$, respectively. Shock-alone presentations were delivered according to the same schedule used during conditioning. All conditioning and postconditioning shock sessions were conducted with the lever retracted. All groups remained in the chamber 6 min after the last US presentation.

Recovery and testing. During each of the next three sessions, all subjects were returned to baseline conditions. Testing was conducted during the subsequent two sessions. The first $20 \mathrm{~min}$ of each test session served as the prepunishment baseline against which the effectiveness of the $\mathrm{CS}$ as a punisher was assessed.

During the 20-min conditioned punishment period, each leverpress produced a 2 -sec presentation of the CS. The punishment contingency was suspended during each CS presentation; i.e., a response during the CS had no effect on its duration. During the first $4 \mathrm{~min}$ of the punishment period, all scheduled reinforcers were cancelled in order to reduce variance caused by uncontrolled effects of reinforcement. At the end of the 4-min interval, reinforcers were available for all rats.

Data analysis. Performance was assessed in terms of suppression ratios in the form B/(A + B) (Annau \& Kamin, 1961); A refers to the number of responses in the prepunishment period (Minutes 1-20), and B refers to the number of responses while the punishment contingency was in effect. Separate suppression ratios were computed for each of four successive 5-min intervals of testing based on the total prepunishment responses divided by four. A suppression ratio of .5 indicates no suppression, whereas a ratio of 0 signifies complete suppression. In computing these suppression ratios for the first 5 -min interval of testing, the first punished response was not included since it did not reflect suppression. A rejection criterion of $p \leqslant .05$ was used for all statistical analyses.

\section{Results and Discussion}

The mean number of prepunishment responses on Test Day 1 for Groups P-0, P-.4, P-1.6, U-0, U-.4, 
and U-1.6 were $708.4,600.5,545.0,633.1,619.1$, and 659.3 , respectively. These means on Test Day 2 were $710.5,551.5,600.0,670.3,612.6$, and 634.0 , respectively. A 2 by 3 (ISI by shock intensity) between-groups analysis of variance with repeated measures over both test days failed to yield any statistically significant effects (all Fs $<1.6$ ).

Figure 1 presents the mean suppression ratios for successive 5-min intervals of testing. As the figure illustrates, the suppression ratios for all three paired groups were lower than those of the three unpaired groups $[F(1,42)=28.09]$. As expected, this result indicates that greater associative strength accrued to the CS in the paired conditions. Extinction of the suppressive effect of the CS was nearly complete for the unpaired groups by the end of Day 1 testing, whereas for the paired groups extinction was not as evident until late in testing on Day 2. This differential rate of extinction resulted in a significant interaction of ISI, Days, and 5-min interval within Days $[F(3,126)=7.62]$.

Of principal importance to Rescorla's analysis of postconditioning exposure to intense shock is the replication of US-inflation effects in the paired condition but not in the unpaired condition. Inspection of Figure 1 shows that Group P-1.6 was more suppressed than Group P-0 almost entirely throughout testing, whereas Groups U-1.6 and U-0 showed little, if any, consistent difference between them. Planned comparisons showed that Groups P-1.6 and P-0 differed significantly $[F(1,42)=4.58]$, whereas Groups U-1.6 and U-0 did not $(F<1)$. The failure to obtain a significant difference between Groups U-1.6 and U-0 is consistent with an associative

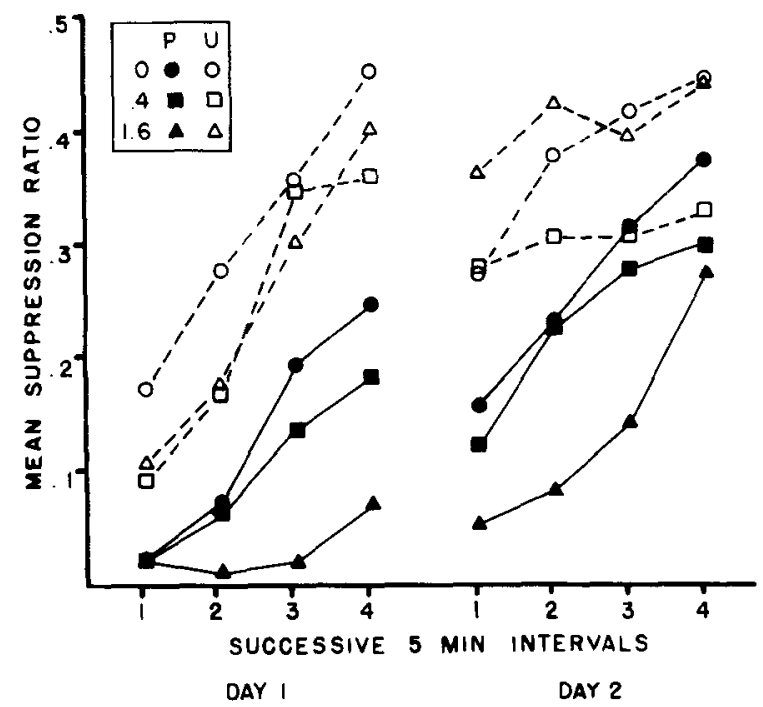

Figure 1. Mean suppression ratios across 5-min intervals of testing for groups receiving paired (P) vs. unpaired (U) CS-US presentations followed by 0 -, .4-, or $1.6-\mathrm{mA}$ shock-alone exposures. interpretation of US-inflation effects.

Inspection of Day 1 of Figure 1 suggests that there was a tendency during Test Intervals 2 and 3 for responding in Group U-1.6 to be more suppressed than in Group U-0. Although these differences were not statistically significant $[F(1,42) \leqslant 1.88]$, they were in the direction obtained with the comparable paired groups. Since relative to the ITI, which averaged $5.5 \mathrm{~min}$, the CS in the unpaired groups was informative (ISI $=2 \mathrm{~min}$ ), this suggestive difference might have been due to weak associative effects. Because of this consideration, and the fact that the use of unpaired control groups is an experimentally novel procedure for assessing postconditioning exposure to an intense shock, a replication of Groups U-0 and U-1.6 was conducted. However, the ISI was increased to $2.75 \mathrm{~min}$. This interval is exactly half of the average ITI, and thus the CS was noninformative.

In this replication, an analysis of the prepunishment responses for Groups U-0 and U-1.6 $(n=8)$ failed to yield any statistically significant effects. The mean suppression ratio scores of Groups U-0 and U-1.6 for successive 5 min of testing on Day 1 were $.24, .43, .45, .47$ and $.27, .37, .41, .43$, respectively. An analysis of these data showed no significant effect of Groups or interaction of Groups by 5 -min interval of testing $(F s<1)$. The overall relative position of these two groups reversed on Day 2 of testing. Although subject to the dangers inherent in accepting the null hypothesis, this replication confirms the absence of a difference between Groups U-0 and U-1.6 in Experiment 1. Since there was a "lesser" degree of differential response suppression among the groups of the replication than among the analogous experimental groups, associative factors may have mediated the tendency toward an inflation effect in Group U-1.6 of Experiment 1.

That postconditioning exposure to intense shock enhanced suppression in the paired treatments only is consistent with Rescorla's (1974) view that (1) the CS and US events are independently represented and that the representation of the US may be manipulated without altering the integrity of their association, and (2) the CR is a consequence of activating the US representation. Within this framework, exposure to intense shock inflated the value of the US representation for both Groups P-1.6 and U-1.6. However, since activation of the US representation is assumed to occur "via an associative connection from the CS memory" (Rescorla, 1974, p. 101), Group U-1.6 did not show significantly more response suppression to the CS than Group U-0 because of the weak, or nonexistent, associative connections between the CS and US. Alternatively, 
Group P-1.6 responding was more suppressed than Group P-0 responding because the association between the CS and US was strong enough to activate the inflated US representation. These data thus corroborate Rescorla's (1974, Experiment 1b) demonstration of postconditioning US-inflation effects and suggest that US-inflation effects are associative.

One aspect of the present data which has not yet been addressed conflicts with Rescorla's (1974) findings. Rescorla observed that a group subsequently exposed to shock-alone presentations of the same intensity used during conditioning showed less suppression than a group which did not receive shock following conditioning. He argued that subsequent exposures to shock "deflated" the value of the US representation via habituation to shock.

As is apparent in Figure 1, Group P-.4 did not show less suppression than Group P-0 but, rather, tended to be more suppressed. An overall comparison of Groups P-.4 and P-0, however, revealed that this difference was within the range of expected random variation $(F<1)$. A similar comparison of Groups U-0 and U-.4 proved nonsignificant as well $[F(1,42)=1.89]$.

The discrepancy between the results of Rescorla's study and the present results may be due to differences in the effective shock intensity of the US. In the present study, the US was a $2-\mathrm{sec}, .4-\mathrm{mA}$ shock, whereas in Rescorla's, it was a .5-sec, .5-mA shock. Church (1969) has demonstrated that longer-duration shock may be functionally equivalent to a more intense shock of shorter duration. It may be that the failure to find habituation to shock in the present study may simply reflect the use of a functionally more intense shock than that used by Rescorla. This interpretation is consistent with the findings of Heth (1976) and Sherman and Maier (1978), who used the same nominal intensity shock as Rescorla (1974) but at longer durations. Heth (Experiment 2) failed to observe any decremental effect of 70 4-sec, .5-mA shock-alone exposures on the conditioned punishing effects of a CS paired with that shock for 10 trials. It made no difference whether shock-alone presentations preceded or followed the 10 CS-US pairings. Similarly, Sherman and Maier (Experiment 3) found no evidence that 80 2-sec, .5-mA shock-alone presentations diminished the effects of a CS subsequently paired with that shock for 20 trials. Aside from the discrepancy described above, the present results are in agreement with those of Rescorla.

\section{EXPERIMENT 2}

Experiment 1 demonstrated that US-inflation effects can be obtained using a 2 -sec trace CS and a conditioned punishment measurement procedure. This US-inflation effect has been taken as evidence that the CS and US were associated during conditioning (see Rescorla, 1977). If the forward relationship of the CS to the US, i.e., its informational value, importantly determines what events enter into association with that CS, exposure to an intense shock following simultaneous conditioning with a moderate shock may not yield US-inflation effects. Experiment 2 examined this possibility.

\section{Subjects}

\section{Method}

The subjects were 36 male albino rats obtained from the Holtzman suppliers in Madison, Wisconsin. The rats were approximately 125-130 days old at the beginning of the experiment, and were maintained at $80 \%$ of their free-feeding weights.

\section{Apparatus}

The experimental equipment was the same as that described in Experiment 1.

\section{Procedure}

All aspects of the procedure were identical to that described in Experiment 1 except as otherwise noted below.

Conditioning and postconditioning US exposure. After leverpress and VI baseline training, the rats were matched for response rates and assigned to one of three groups $(n=12)$. During conditioning, two of the groups received paired (P) CS-US presentations with an ISI of $0 \mathrm{sec}$, i.e., simultaneous conditioning, whereas the third group received unpaired (U) CS-US presentations with an ISI of $120 \mathrm{sec}$. The latter group provided a reference against which associative effects of the simultaneous procedure might be assessed in the same manner as that used to assess the associative effects of the trace conditioning procedures of Experiment 1 . In all, 16 CS-US presentations were given over 2 successive days.

During each of the next two sessions, all groups received eight shock-alone exposures. For the unpaired group (U-0) and one of the paired groups (P-0), shock intensity was $0 \mathrm{~mA}$, i.e., no shock was presented. However, for the other paired group (P-1.6), shock intensity was $1.6 \mathrm{~mA}$. Subsequent recovery of baseline, testing, and data analysis followed the procedure of Experiment 1.

\section{Results and Discussion}

The mean number of prepunishment responses on Test Day 1 for Groups P-0, P-1.6, and U-0 were $551.3,584.7$, and 550.9, respectively. On Test Day 2, these respective means were 543.3613 .2 , and 553.9. A repeated measures (Day 1 and 2 ) one-way analysis of these data failed to yield any statistically significant effects (all Fs $<1$ ).

Figure 2 presents the mean suppression ratios for Groups P-0, P-1.6, and U-0 for successive 5-min intervals for both test days. However, since group differences were largely attenuated on Day 2, statistical analyses and discussion are limited to Day 1 performance. As is apparent in the figure, the three groups differed in the degree of suppression produced by the $\operatorname{CS}[F(2,33)=8.18]$. Moreover, the differences among the groups interacted with the interval of testing $[F(6,99)=2.63]$. Consistent with 


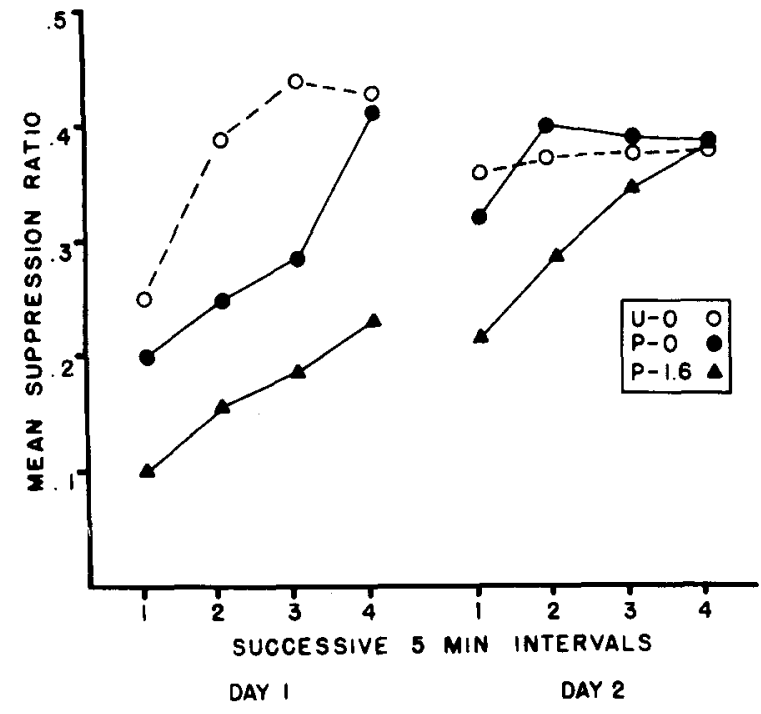

Figure 2. Mean suppression ratios across 5-min intervals of testing for groups receiving simultaneously paired (P) or unpaired (U) CS-US presentations followed by either $0-$ or $1.6-\mathrm{mA}$ shockalone exposures.

this interaction, Groups $\mathrm{U}-0$ and P-0 differed significantly only during Test Intervals 2 and 3 $[\mathrm{Fs}(1,33) \geqslant 5.12]$, although the comparison of the overall mean difference between Groups $\mathrm{I}-0$ and $\mathrm{P}-\mathrm{0}$ attained marginal significance $[F(1,33)=3.21, .05$ $<\mathrm{p}<.10]$. This result is consistent with previous demonstrations showing that simultaneous fear conditioning yields greater conditioning than a variety of nonassociative control procedures (Heth \& Rescorla, 1973; Mahoney \& Ayres, 1976; Sherman \& Maier, 1978).

Of primary interest is the relative degree of suppression exhibited by the simultaneously conditioned group that received postconditioning to intense shock, Group P-1.6. As the figure illustrates, Group P-1.6 showed more initial and more sustained suppression than did Group P-0. A planned comparison between the overall means of Groups P-1.6 and $P-0$ revealed a significant difference $[F(1,33)$ $=5.04]$.

The results of this experiment suggest that USinflation effects can be obtained following simultaneous conditioning; postconditioning exposure to intense shock enhanced the suppressive effects of the simultaneously conditioned CS. This result is consistent with similar manipulations obtained following first-order trace (Experiment 1) and delay (Rescorla, 1974) conditioning procedures.

\section{GENERAL DISCUSSION}

Experiment 1 demonstrated that exposure to intense shock alone, following trace conditioning trials with a moderate shock US, only produced enhanced suppression to the CS when the CS and US shared a relatively strong degree of associative strength. The degree of acquired associative strength was manipulated by varying the ISI between the 2 -sec CS and US. At ISIs of 2 and $2.75 \mathrm{~min}$, in which the CS and US were, at best, weakly associated, little or no effect of postconditioning exposure to intense shock was obtained. However, following conditioning with a 10-sec ISI in which the CS and US were strongly associated, exposure to intense shock enhanced the suppressive effect of the CS. Thus, the demonstration that associative effects must mediate the enhanced suppression to the CS precludes explanations which argue that exposure to intense shock may have simply produced greater suppression to the CS because of sensitization and/or increases in motivational level (Kimble, Mann, \& Dufort, 1955). Rescorla (1974) did not directly assess whether postconditioning US-inflation manipulations were, in fact, associative. In this way, these results increase the validity of Rescorla's interpretation of the effects of postconditioning exposures to intense shock in terms of inflating the US representation.

In view of the theoretical significance of Rescorla's demonstrations of US-inflation effects, it is of considerable consequence to extend the empirical generality of that phenomenon. The present experiments differed from Rescorla's procedure in a number of ways: CS duration, ISI, intertrial interval, US intensity and duration, and technique for assessing the associative relationship between the CS and US. Additionally, in Rescorla's procedure, the presentations of postconditioning shocks were preceded by a signal in order to block potential conditioning to contextual cues, whereas in the present experiments no such signal was employed. That essentially similar results were still obtained is consistent with a conceptually similar experiment in which signaled or unsignaled US presentations were found to be equally effective in producing reinstatement of the suppressive effects of a previously extinguished CS (Rescorla \& Heth, 1975).

However, of primary interest in the present investigation was whether or not different learning (S-S or S-R) mediates conditioning obtained with simultaneous and forward procedures. Experiment 2 provided evidence that, analogous to first-order forward conditioning, simultaneous conditioning is also a product of S-S learning. Consequently, in terms of temporal priority to the US, the informational character of the CS does not influence the qualitative nature of the events entering into association with the CS, at least as measured by the US-inflation technique.

Rescorla (1973) suggested that US-inflation effects may be casually interpreted as indicating that "the subject may learn it was presented a painful stim- 
ulus after the CS [italics mine]" (p. 143). That US-inflation effects may be obtained with a simultaneous CS suggests that it may be sufficient to say that the subject may learn it was presented a painful stimulus with the CS. The role of ISI in securing the representation of the CS-US association may be independent of its effect on the mechanism which evokes the CR. That is, once the CS-US association is represented, production of the conditioned fear response may simply be a consequence of evoking the representation of the US memory by the CS. The temporal relationship between the CS-US events may not be represented with the CS-US memory once they are associated. This notion has been expressed in the process model of Wagner and Terry (1975). In their model, "conjoint rehearsal" of the CS-US events yields excitatory conditioning independently of their temporal sequencing.

It is clear that informational theories of classical conditioning will have to confront the increasing number of demonstrations showing the basic comparability of simultaneous and forward fear conditioning (Heth \& Rescorla, 1973; Mahoney \& Ayres, 1976; Sherman \& Maier, 1978). The present study complements these findings by providing evidence that forward (Experiment 1) and simultaneous (Experiment 2) conditioning, as reflected by the US-inflation technique, are both mediated by S-S learning. These findings provide general support for the recent reformulation of the effects of "informational" variables in terms of a contiguity-based model of Pavlovian conditioning (Rescorla \& Wagner, 1972).

\section{REFERENCES}

Annau, Z., \& Kamin, L. J. The conditioned emotional response as a function of US intensity. Journal of Comparative and Physiological Psychology, 1961, 54, 428-432.

Catania, A. C., \& Reynolds, G. S. A quantitative analysis of the responding maintained by interval schedules of reinforcement. Journal of the Experimental Analysis of Behavior, 1968, 11, 327-383.

ChuRCh, R. M. Response suppression. In B. A. Campbell \& R. M. Church (Eds.), Punishment and aversive behavior. New York: Appleton-Century-Crofts, 1969. Pp. 111-156.

EGGER, M. D., \& Miller, N. E. Secondary reinforcement in rats as a function of information value and reliability of the stimulus. Journal of Experimental Psychology, 1962, 64, 97-104.
Heth, D. C. Simultaneous and backward fear conditioning as a function of number of CS-US pairings. Joumal of Experimental Psychology: Animal Behavior Processes, 1976, 2, 117-129.

Heth, D. C., \& Rescorla, R. A. Simultaneous and backward fear conditioning in the rat. Journal of Comparative and Physiological Psychology, 1973, 82, 434-443.

Kamin, L. J. Predictability, surprise, attenion, and conditioning. In B. A. Campbell \& R. M. Church (Eds.), Punishment and aversive behavior. New York: Appleton-Century-Crofts, 1969. Pp. 279-296.

Kimble, G. A., Mann, L. I., \& Dufort, R. H. Classical and instrumental eyelid conditioning. Joumal of Experimental Psychology, 1955, 49, 407-417.

Mackintosh, N. J. Stimulus selection: Learning to ignore stimuli that predict no change in reinforcement. In R. A. Hinde \& J. Stevenson Hinde (Eds.), Constraints on learning. London: Academic Press, 1973. Pp. 75-96.

Mackintosh, N. J. A theory of attention: Variations in the associability of stimuli with reinforcement. Psychological Review, 1975, 82, 276-298.

Mahoney, W. J., \& Ayres, J. J. B. One-trial simultaneous and backward fear conditioning as reflected in conditioned suppression of licking in rats. Animal Learning \& Behavior, 1976, 4, 357-362.

Rescorla, R. A. Informational variables in Pavlovian conditioning. In G. H. Bower (Ed.), The psychology of learning and motivation: Advances in research and theory. New York: Academic Press, 1972, Pp. 1-46.

Rescorla, R. A. Effect of US habituation following conditioning. Journal of Comparative and Physiological Psychology, 1973, 82, 137-143.

RESCORLA, R. A. Effect of inflation of the unconditioned stimulus value following conditioning. Journal of Comparative and Physiological Psychology, 1974, 86, 101-106.

Rescorla, R. A. Pavlovian second-order conditioning: Some implications for instrumental behavior. In H. Davis \& H. M. B. Hurwitz (Eds.), Operant-Pavlovian interactions. New York: Erlbaum, 1977. Pp. 133-164.

Rescorla, R. A., \& Heth, D. C. Reinstatement of fear to an extinguished conditioned stimulus. Journal of Experimental Psychology: Animal Behavior Processes, 1975, 104, 88-96.

Rescorla, R. A., \& WAgNeR, A. R. A theory of Pavlovian conditioning: Variations in the effectiveness of reinforcement and nonreinforcement. In A. H. Black \& W. F. Prokasy (Eds.), Classical Conditioning II: Current theory and research. New York: Appleton-Century-Crofts, 1972.

Rozeвоoм, W. W. "What is learned?"-An empirical enigma. Psychological Review, 1958, 65, 22-33.

Sherman, J. E., \& Maier, S. F. The decrement in conditioned fear with increased trials of simultaneous conditioning is not specific to the simultaneous procedure. Learning and Motivation, 1978, 9, 31-53.

WAGNER, A. R., \& TERRY, W. S. Backward conditioning to a CS following an expected vs. surprising UCS. Animal Learning \& Behavior, 1975, 3, 370-374.

(Received for publication September 29, 1977; revision accepted June 15,1978 .) 